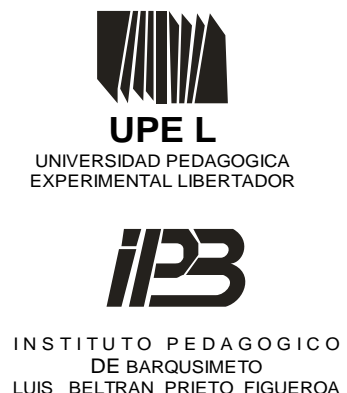

LUIS BELTRAN PRIETO FIGUEROA

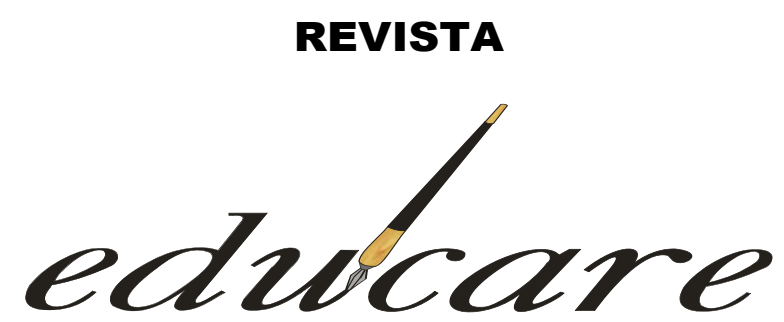

Órgano Divulgativo de la Subdirección de Investigación y Postgrado del Instituto Pedagógico de Barquisimeto “Luis Beltrán Prieto

\author{
BARQUISIMETO - EDO. LARA - VENEZUELA \\ NUEVA ETAPA \\ FORMATO ELECTRÒNICO \\ DEPOSITOLEGAL: ppi201002LA3674 \\ ISSN: 2244-7296
}

Volumen 20 № 1

Enero - Abril 2016

\title{
HABILIDADES SOCIALES PARA EL DESARROLLO DE UNA CULTURA DE PAZ
}

\section{SOCIAL SKILLS FOR THE CULTURE OF PEACE DEVELOPMENT}

\author{
Yonis Eduardo Romero Torrealba*
}

\section{* UNIVERSIDAD PEDAGÓGICA EXPERIMENTAL LIBERTADOR INSTITUTO PEDAGÓGICO BARQUISIMETO}




\section{HABILIDADES SOCIALES PARA EL DESARROLLO DE UNA CULTURA DE PAZ}

SOCIAL SKILLS FOR THE CULTURE OF PEACE DEVELOPMENT

\section{TRABAJO DE INVESTIGACIÒN}

Recibido: 27-01-16

\author{
Yonis Eduardo Romero Torrealba * \\ UPEL-IPB
}

Aceptado:11-04-16

\section{RESUMEN}

Las habilidades sociales son conductas que facilitan la relación interpersonal entre los seres humanos desde la comprensión y el respeto aspirando orientar la acción de las personas en la sociedad. El propósito de esta investigación fue conocer desde los haceres de la comunidad educativa, sus creencias y opiniones sobre la necesidad de construir una cultura de paz. La naturaleza cualitativa de la investigación se orientó a desarrollar una metodología interpretativa mediante la observación y la entrevista, que permitió apreciar una estructura de la realidad desde la cual se reporta como hallazgo la siguiente reflexión: La búsqueda de la unidad por medio del diálogo como alternativa para resolver los conflictos, con interés por una mejor calidad de vida y aprender a relacionarse sin extraviar la integridad personal con la colaboración de todos construye una cultura de paz.

Descriptores: cultura de paz, habilidades sociales, comunidad educativa

\begin{abstract}
Social skills are behaviors that facilitate the interpersonal relationship between human beings from understanding and respect aiming to guide the action of people in society. The purpose of this research was to learn from the actions of the educational community, their beliefs and opinions about the need to build a culture of peace. The qualitative nature of the research was oriented to develop an interpretative methodology through observation and interview, which allowed to appreciate a structure of reality from which the following reflection is reported as a finding: The search for unity through dialogue as an alternative to resolve conflicts, with an interest in a better quality of life and learn to relate without losing personal integrity with everybody's collaboration builds a culture of peace.
\end{abstract}

Keywords: culture of peace, social abilities, educational community

*Profesor Especialista en Mecánica Industrial. Magister en Educación Mención Investigación Educacional. yonis.eduardo@gmail.com 


\section{INTRODUCCIÓN}

La situación actual de las relaciones humanas en que nos encontramos está en condiciones de urgencia, donde es necesario tomar las riendas e intervenir, para encontrar una salida que nos asegure una sensación de bienestar colectivo y seguridad individual frente a la gran ola de violencia que nos está abatiendo. No se trata de solo casos en las escuelas, es un malestar global, que se viene desatando desde hace ya algunas décadas y, para corroborar esto, ya Goleman (1995) nos advertía que: "Vivimos en una época en la que el tejido de la sociedad parece deshacerse a una velocidad cada vez mayor, en la que el egoísmo, la violencia y la ruindad espiritual parecen corromper la calidad de nuestra vida comunitaria." (p. 16)

Luego de algunos años se confirma la predicción que Goleman nos había hecho, puesto que, ningún sujeto puede cerrar los ojos ante tantos problemas de índole social y que repercuten en el carácter particular del quehacer de los individuos, haciendo de la vida diaria un hecho de supervivencia más que de disfrute pleno y felicidad social.

Este panorama que se presenta ante nosotros como ciudadanos es bastante crucial, donde cada vez se presentan más casos de violencia, agresividad, destrucción y autodestrucción, como también, de sumisión y pasividad, observado tanto desde la perspectiva del individuo como del colectivo; de ingobernabilidad dado a los innumerables incumplimientos de las normas y además la pérdida de los principios y valores morales cuando en su defecto se aprecia el egocentrismo y la falta de sentido de pertenencia y de participación.

Por tal motivo, se debe enfrentar esta realidad desde la escuela, que dado su rol social pueda contribuir al fomento de una cultura de paz en todos los sujetos que por ella circundan, pues, la escuela también está sometida por los mismos problemas sociales que existen fuera de ella, debido a que siendo parte de la sociedad se ven reflejados considerablemente sus problemas.

Así pues, cada problema, cada suceso donde exista violencia, se convierte en un acontecimiento y la misma está latente en los individuos esperando la ocasión para ser drenada. Es necesario tener en cuenta, que dependiendo del contexto donde se ubica la institución educativa, se podrán apreciar características esenciales que las diferencian de otras, como no está de más decir que en ocasiones los valores y actitudes del conflicto que se encuentran presentes en el contexto pueden ser más influyentes que aquellos que intenta transmitir la escuela a través de sus programas y planificaciones. 
Debe quedar por sentado, que para que se dé una cultura de paz, es necesario que exista un fuerte vínculo entre la escuela, la comunidad, los padres y los representantes. Pues en la familia, en la comunidad y en las escuelas existen múltiples oportunidades para realizar intercambios comunicativos entre sus integrantes, los cuales pueden realizarse con éxito si entre los sujetos vinculados existen, así ellos mismos lo ignoren, algunas habilidades sociales. Por eso se habla de habilidades sociales como todas aquellas destrezas que nos sirven para relacionarnos con los demás, una capacidad para lograr objetivos interpersonales manteniendo la autoestima sin dañar a las personas que le rodean y un camino que puede conducir a la paz social.

\section{Propósitos del estudio}

Conocer desde los haceres de la comunidad educativa, sus creencias y opiniones sobre la necesidad de construir una cultura de paz.

Interpretar los significados que emergen de las habilidades sociales en los haceres de los integrantes de la comunidad educativa para la construcción de una cultura de paz.

\section{ARGUMENTACIÓN TEÓRICA}

\section{La cultura de paz}

El hombre es un ser social que se encuentra relacionándose de manera continua, participando constantemente en un intercambio de experiencias, en eventos y situaciones, donde se pueden presentar conflictos debido a la diversidad de opiniones y formas de concebir al mundo. En una mirada al curso de la historia podemos observar que, al llevar a cabo sus ideas, el hombre, en oposición a las de otro que no está dispuesto a ceder, ha recurrido a la violencia como forma de coerción. Esto genera como consecuencia, un clima social en donde no es posible desarrollar ni emitir las ideas con libertad. Actualmente, este fenómeno de violencia es común en nuestra sociedad, donde para resolver un conflicto se propone como alternativa frecuente recurrir al enfrentamiento, empleando la fuerza y reprimiendo las ideas de quienes se oponen.

De tal manera, ha surgido la necesidad de extender por doquiera que el hombre se encuentre la idea de una cultura de paz en oposición a la violencia, para crear un ambiente 
pleno, positivo y armónico, en donde todos los hombres podamos desarrollar nuestras ideas sin temor a ser maltratados, es decir, donde se pueda ser y convivir.

Por esa razón, podemos decir que la paz es un proceso dinámico y continuo de participación integral del individuo con la sociedad, el cual sus principios y valores generan actitudes y orientan sus acciones por medio de la comunicación que se dirige hacia la resolución de conflictos en rechazo de la violencia. Explica González (2009) que la paz “(...) aspira orientar la acción de las personas en la sociedad convirtiéndose en una máxima universal que se apoya en esa búsqueda de felicidad personal y desarrollo social que se evidencia a lo largo de la historia". (p.23). En vista a ese motivo, debido al desbordamiento de la violencia directa, física, psicológica y estructural, consecuencia de la insatisfacción de las necesidades básicas y producto de patrones culturales aprendidos, es evidente la necesidad de fomentar una cultura de paz que devuelva al hombre y a quienes le rodean la tranquilidad de desenvolverse en cualesquiera de las esferas de la sociedad, lo cual, supone modificar patrones negativos de conducta y contrarrestar sus efectos por medio de la educación, "puesto que las guerras nacen en la mente de los hombres, es en la mente de los hombres donde deben erigirse los baluartes de la paz" según criterio de la UNESCO (2002)

\section{El quehacer de la comunidad educativa}

De tal manera, la comunidad educativa en su quehacer debe intervenir de manera individual y/o colectiva en la gestión educativa para el desarrollo de la cultura de paz necesaria que demanda la situación actual de las relaciones humanas entre los participantes de nuestra sociedad, como lo explica Molano (2011) "para realizar modificaciones estructurales que conviertan a las personas en agentes activos de transformación y así favorecer la mejora de las relaciones, creando un clima positivo en el que los conflictos sean una oportunidad para el diálogo constructivo". (p.60)

Es así que, desde la escuela se debe debatir y organizar las ideas que a través de una política educativa en pro de la cultura de paz sea posible introducir dentro de las instalaciones un clima armónico y próspero, no solo para facilitar los procesos de aprendizaje, sino que también puedan rescatarse y construirse los valores indispensables para la sana y plena convivencia, a través del desarrollo de habilidades sociales. 


\section{Las habilidades sociales}

Con las habilidades sociales se busca, según Buxarrais (1997), "el desarrollo de los comportamientos y las actitudes prosociales que permitan enfrentarse de forma positiva a los problemas sociales y de carácter personal, aumentando la propia autoestima y mejorando las relaciones con los demás" (p. 139). Así como también, se toma en cuenta la definición de habilidades sociales de Yegres (1999), la cual nos explica que:

Son el conjunto de comportamientos interpersonales que va aprendiendo la persona y que configuran su competencia social en los diferentes ámbitos de relación. Tales comportamientos pretenden modificar el entorno social de vida de modo que se obtengan consecuencias deseadas y se eviten o supriman hechos o situaciones no deseables. (p. 176)

Por otra parte, Morales (2007) explica que "las habilidades sociales son conductas que facilitan la relación interpersonal, de forma no agresiva ni inhibida, sino asertiva" (p. 22). De tal manera, las habilidades sociales se refieren a la manera en cómo el sujeto interactúa con sus semejantes, la cual puede observarse y describirse $\mathrm{y}$, en la medida en que las posea, puede desenvolverse de manera más efectiva para llevar a cabo lo que se proponga en un medio donde tenga que relacionarse con otros individuos.

\section{ASPECTOS METODOLÓGICOS}

\section{Naturaleza de la investigación}

Para presente estudio, por estar centrado en la misma experiencia humana, los acontecimientos son comprendidos de manera objetiva si son observados desde adentro, inmiscuyéndose en la realidad y la perspectiva de los sujetos. Para tal fin, se asume una investigación cualitativa, mediante la cual se aprecia la calidad de las actividades, relaciones, asuntos, medios, materiales o instrumentos en una determinada situación o problema, analizando exhaustivamente y con sumo detalle un asunto o actividad en particular.

De manera que, la investigación cualitativa brinda la posibilidad de conocer el mundo desde la perspectiva de los sujetos, en tanto sea una representación de las vivencias que se van logrando en su medio natural, Martínez (2006) explica que "trata de identificar, básicamente, la naturaleza profunda de las realidades, su estructura dinámica, aquella que da razón plena de su comportamiento y manifestaciones". (p.66). 
La investigación cualitativa se orienta hacia la compresión de la realidad social y cultural como un todo unificado, se indaga científicamente la subjetividad, las relaciones intersubjetivas, los saberes, los valores, los significados, las creencias y los comportamientos que subyacen en las acciones sociales y humanas, individuales y colectivas de los propios actores sociales, que se develan en las continuas interacciones que se ejecutan obedeciendo a los significados latentes en la naturaleza compartida, llevada a cabo mediante el lenguaje, la comunicación y la cultura.

\section{Diseño}

Con los datos que se tomaron en el momento del abordaje de la realidad, enriquecidos de las ideas y pensamientos asomados en los diálogos realizados, se procedió a la búsqueda de sus significados, para captar la estructura de la realidad del estudio. En esta búsqueda, los significados se hallaron en los datos mediante la interpretación entendida como "la captación o comprensión de una vida psíquica por otra vida psíquica diferente de la primera" (p. 121), según Martínez (1996). De manera que la realidad emergió de los datos en medio de un descubrimiento de las intenciones y los significados de la acción de los actores sociales, tomando en cuenta la función que dicha acción desempeña en sus vivencias y el nivel de condicionamiento ambiental y cultural que lo determina.

Se observó a los actores sociales que al relacionarse entre sí, con múltiples intercambios de acciones en un empleo de la comunicación que conocen, construyen la estructura subjetiva que comparten y que los identifica. Teniendo en cuenta que los datos son los significados que emergen de un momento interpretativo, sobre el conjunto de ideas y pensamientos que se construyen en el razonamiento del investigador al momento del abordaje de la realidad y el develamiento de los datos existentes, se les suspendieron los presupuestos que contenidos mediante una reducción subjetiva, de manera que puedan observarse los fenómenos tal cual como son en sí mismos.

\section{Contexto y muestra}

La investigación fue realizada en el Liceo Bolivariano Dr. "Rafael Villavicencio ubicado en Barquisimeto, estado Lara, Venezuela. 
La selección de los actores sociales se realizó bajo carácter intencional, donde los mismos fueron elegidos de acuerdo a la direccionalidad que se plantea en la investigación. Según Martínez (2008) se considera "una muestra que sea comprehensiva y que tenga, a su vez, en cuenta los casos negativos o deviantes, pero haciendo énfasis en los casos más representativos y paradigmáticos" (p.148) además conforman un grupo social con características similares en su organización.

Los actores sociales considerados en una investigación cualitativa, de acuerdo con Pérez Serrano (1998), se eligen porque cumplen ciertos requisitos en el mismo contexto educativo o en la misma población, por esa razón, la selección deliberada e intencional de las personas o grupos no se realiza al azar para completar una muestra de tamaño, más bien se toman uno a uno de acuerdo con el grado en que ajustan a los criterios o atributos establecidos por el investigador.

Como se puede apreciar en el cuadro $\mathrm{N}^{\circ} 1$ se eligió intencionalmente a dos (2) actores sociales los cuales fueron estudiantes pertenecientes al nivel de quinto año y un (1) actor social docente-representante, los cuales para el momento de la investigación estaban haciendo vida en la comunidad educativa, siendo pertenecientes a la misma.

Cuadro $\mathrm{N}^{\circ} 1$.

Muestra intencional de los actores sociales

\begin{tabular}{l|l}
\hline ACTOR SOCIAL & CÓDIGO \\
\hline Estudiante 01 & $\mathrm{eE01}$ \\
\hline Estudiante 02 & $\mathrm{eE02}$ \\
\hline Docente-Representante & eDR01 \\
\hline
\end{tabular}

\section{Descripción de las técnicas e instrumentos para la recolección de la información}

Las técnicas de recolección de información elegidas para llevar a cabo el estudio, acordes con la naturaleza de su realidad, son la observación participativa y la entrevista semiestructurada. En cuanto a la observación, esta es necesaria para toda investigación, pues es el vehículo por el cual nos apropiamos de la realidad. Es necesario aclarar que todos los sujetos observan y, por ende, también el investigador. Dados estos argumentos se empleará la 
observación participativa, pues, el investigador (o sujeto cognoscente) al formar parte del entorno, comparte las vivencias y conocimientos de los sujetos que ha elegido con la orientación de su estudio. El registro de las observaciones y su posterior revisión proporciona los elementos necesarios para la realización de la reducción subjetiva.

En cuanto a la entrevista, ésta es un encuentro donde se intercambian y unifican las perspectivas de los sujetos (sus observaciones), y de cuyo producto, se obtiene una perspectiva enriquecida (de la suma o de la resta) que está más cercana de la verdad. Ciertamente, todos los sujetos tienen conocimientos sobre un tema en particular, sin embargo, se denomina investigador a quién ha demostrado interés hacia el tema particular de investigación, y por lo tanto, coordina el fluir de los conocimientos.

Es así como el conocimiento del otro, de su personalidad, de su individualidad en medio de todo, toma forma a través de la entrevista, donde se proporcionan conocimientos, por medio de la comunicación verbal y no verbal, en el momento del encuentro, y posteriormente, cuando sea necesario volver a observar los datos con mayor claridad.

La intersubjetividad de un encuentro, o entrevista, debe tenerse presente para: "aclarar términos, descubrir ambigüedades, definir los problemas, orientar hacia una perspectiva, patentizar presupuestos y las intenciones, evidenciar la irracionalidad de una proposición, ofrecer criterios de juicio o recordar los hechos necesarios" (p. 131), según lo expresado por Martínez (1996); así como también, por razón de ser sujetos emocionales: "motivar al interlocutor, elevar su nivel de interés y colaboración, reconocer sus logros, prevenir una falsificación, reducir los formalismos, estimular su memoria, aminorar la confusión” (p. 132). Además, se debe tener presente que en un encuentro existe una toma de decisiones.

Una vez que nos sumergimos en la realidad, se elaboró la descripción protocolar como instrumento donde se muestran los sentidos que emergen de la subjetividad de los actores sociales, mediante un abordaje interpretativo y manteniendo en paréntesis los prejuicios a partir del empleo de una reducción subjetiva.

Técnicas para el análisis y sistematización de la información: categorización, codificación, triangulación, estructuración y contrastación

A la información proporcionada por los actores sociales, desde la cual emergieron los datos que se cristalizaron en el contenido de la descripción protocolar, se les asignó símbolos 
verbales o categorías que cumplieron la función de expresiones para describir los atributos de los fenómenos observados, tal como son presentados en el cuadro $\mathrm{N}^{\circ} 2$ a continuación.

Cuadro $\mathrm{N}^{\circ} 2$.

Categorías, dimensiones y su código

\begin{tabular}{l|l|l}
\hline CATEGORÍAS & DIMENSIONES & CÓDIGO \\
\hline \multirow{2}{*}{ Paz } & Paz & P00 \\
\cline { 2 - 3 } & Valores & Va00 \\
\cline { 2 - 3 } Violencia y agresividad & Tolerancia & T00 \\
\hline \multirow{2}{*}{ Contexto educativo } & Violencia y agresividad & V00 \\
\hline \multirow{2}{*}{ Habilidades sociales } & Contexto educativo & C00 \\
\cline { 2 - 3 } & Familia & F00 \\
\cline { 2 - 3 } & Labor docente & Ld00 \\
\hline Intereses & Habilidades sociales & G00 \\
\hline & Gerencia & L00 \\
\cline { 2 - 3 } & Liderazgo & I00 \\
\hline
\end{tabular}

Luego, en un proceso integrador de las categorías y la triangulación de las perspectivas de los actores sociales, por medio del enfoque en los nexos y las relaciones existentes, de la manera sugerida por Martínez (2006) en "un movimiento en espiral, del todo a las partes y de las partes al todo, aumentando en cada vuelta el nivel de profundidad y comprensión” (p. 275), se obtuvo la estructura de la realidad abordada.

Para el enriquecimiento del cuerpo de conocimientos, se procedió a una etapa de contrastación intersubjetiva, que consistió en relacionar, comparar y contraponer los resultados obtenidos acerca de la estructura de la realidad observada, con las presentadas en investigaciones anteriores y teorías diseñadas por los autores pertinentes, con el propósito de proporcionar un momento para establecer las semejanzas y diferencias entre las diversas estructuras que se plantean y la estructura emergente.

Como resultado, la estructura de la realidad del entorno cultural estudiado, ordena los fenómenos observados y representa, como lo explica Martínez (2006), "un contexto, un 
horizonte que dará significado y ayudará a comprender muchas acciones y conductas humanas que, de otra manera resultarían indescifrables" (p.121), es decir, la forma en que el contexto con su carga cultural de valores, ideas y pensamientos influye en el desenvolvimiento de los sujetos.

\section{HALLAZGOS}

Sobre la cultura de paz y su antagónico: cultura de la agresividad, violencia e inseguridad

A propósito de la cultura de paz, las actividades que desarrolla el hombre dentro de un entorno pleno en paz son más humanas $\mathrm{y}$, su desenvolvimiento es de mayor provecho tanto individual como socialmente. Por tal motivo, cuando existe paz, las personas tienen una buena relación entre ellas, pues ésta es una condición intrínseca que genera una mejor calidad de vida. Asimismo, la cultura de paz se promueve por el hombre cuando este se encuentra en relación de contacto con su entorno, se sustenta y alimenta con su actitud y su continuo accionar, de manera individual y social, como también es necesario que el resto los integrantes de la comunidad se desenvuelvan del mismo modo, pues la paz solo es posible con la colaboración de todos.

Sin embargo, el estado actual de las relaciones interpersonales que atentan contra la cultura de paz es consecuencia de las diferentes experiencias que se viven a diario por cada uno de los sujetos que integran nuestra sociedad y, dan pie al diseño de prejuicios acerca del otro como mecanismos de defensa de la integridad personal. Hasta tal punto que, la cultura de paz está siendo vulnerada desde parte importante de los hogares, con la transmisión de una diversa especie de mensajes de odio, resentimiento, desdicha y desconfianza entre otros. Por esa razón, tanto en la comunidad educativa como en sus adyacencias dentro del entorno social, no existe una cultura de paz propiamente dicha, y más bien, puede apreciarse un sentir de estar de "manos atadas" ante esta realidad, donde podemos encontrar agresividad, violencia e inseguridad.

A propósito de la agresividad, Gil-Verona (2002) explica que la agresividad "indica la actitud o inclinación que siente una persona o un colectivo humano a realizar actos violentos" (p.294), los cuales, según lo observado dentro de la comunidad educativa están latentes, manifestándose como focos de violencia integrados por sujetos que ejecutan acciones 
contrarias a la cultura de paz y, que además de esto obtienen apoyo, dado que la misma actitud está reforzada por las condiciones sociales del entorno.

A propósito de la violencia e inseguridad, claro está que para una buena parte de los integrantes de la comunidad educativa, la violencia es desagradable, entendida como lo explica la Organización Mundial para la Salud (2002):

El uso intencional de la fuerza o el poder físico, de hecho o como amenaza, contra uno mismo, otra persona o un grupo o comunidad, que cause o tenga muchas probabilidades de causar lesiones, muerte, daños psicológicos, trastornos del desarrollo o privaciones. (p.1)

Pero la misma se escapa de las manos, por lo difícil que puede ser de controlar al momento que se desata, cuando más bien, se alimenta de manera recíproca, haciéndose cada vez más agravante y perjudicial y cediendo solo por motivos de fuerza o poder. El mecanismo está en que la violencia es una alternativa común para resolver conflictos, dejando a un lado la comunicación y el diálogo en la búsqueda de acuerdos, en cambio se deteriora la paz por medio de la transmisión de mensajes de odio consecuentes. La UNICEF (2002) explica que la violencia "se ejerce con el objeto de intentar dominar la voluntad de una persona, para hacer que se pliegue al poder, a la autoridad, al modo de ser de otra". (p.9)

De tal manera, podemos observar que la agresividad, la violencia y la inseguridad presentes en la comunidad educativa están desbordándose, perjudicando a todos los integrantes que hacen vida en ella y, donde cualquier situación puede vulnerar la paz momentánea y, estos problemas que se presentan se convierten en acontecimientos que deterioran la tranquilidad del día a día. En cuanto a las políticas deben tomar para alcanzar la plenitud y el bienestar que proporciona una cultura de paz dentro de la comunidad educativa, están la búsqueda de unidad por medio del diálogo y la comunicación como alternativa para resolver los conflictos, siempre y cuando se tenga presente y se persiga una mejor calidad de vida; además de aprender a relacionarse sin extraviar la integridad personal, tomando en cuenta la tolerancia como un camino a seguir con la colaboración de todos, y que a pesar que la estructura cultural de determinados grupos no les permite a sus integrantes ceder al establecimiento de la cultura de paz que se persigue, promover los valores considerando que la paz debe ser una condición intrínseca y tácita entre las personas.

Es necesario que para esto se debe ceder un espacio, tomando en cuenta que el vínculo educativo es un vínculo social, en el cual los integrantes de la comunidad educativa 
oponiéndose al desajuste de la cultura de paz, con su actitud se conduzcan haciéndola significativa y estableciendo las bases necesarias para impulsar este desarrollo.

\section{Sobre la familia, la labor docente y el contexto educativo: la tolerancia y otros valores}

A propósito de la familia, su participación y su apoyo es fundamental para el desarrollo de una cultura de paz y la misma debe promover la formación de todos los ciudadanos, empezando por sus hijos. Fabelo (2004) explica que:

Debido a la fuerte presencia que tiene la familia en la educación más temprana del niño, su papel es extraordinariamente importante en la configuración del mundo de valores de esa conciencia en formación. La función que en este sentido juega la familia es en realidad insustituible. Esos valores adquiridos en edades tempranas quedan casi siempre más arraigados en la estructura de la personalidad, lo cual hace más difícil su cambio. (p.148)

A pesar que la familia tiene un rol que es determinante para el desarrollo de una cultura de paz, es necesario tomar en cuenta que nuestra sociedad está cultivando hogares disfuncionales, los cuales afectan a los hijos y de alguna manera los mismos proyectan en todos los escenarios en donde se desenvuelven las vivencias que experimentan dentro del entorno familiar. Fabelo (2004) nos explica que "en muchas ocasiones los padres no tiene plena conciencia de la gran responsabilidad que recae sobre ellos en lo atinente a la educación valorativa de sus hijos o, simplemente, no están lo suficientemente preparados para asumirla" (p.148).

A propósito de la labor docente, entendiendo que el docente y sus estudiantes pertenecen a la misma comunidad estando en relación tanto dentro y fuera del momento en que ocurre la praxis educativa, la labor del docente es el deber de contribuir en la formación de los futuros ciudadanos, tomando en cuenta el cumplimiento de las responsabilidades, el buen trato y la comunicación por su parte y la de todos los integrantes de la comunidad educativa; es así como la labor docente se retribuye de una manera social, puesto que la construcción de una cultura de paz es un beneficio para todos y es necesario que con su acción se proyecte la moral y la ética.

Sin embargo, el modelaje no se está llevando a cabo, más bien los estudiantes observan otros modelos aparte del docente, a los cuales es posible que le presten mayor atención y los mismos no son cónsonos a la cultura de paz. 
A propósito del contexto educativo, en cuanto a cultura de paz se refiere, parte considerable de las instituciones que integran nuestra sociedad no ejercen una influencia positiva para la resolución de los conflictos que se presentan en la comunidad donde reside. Se observa que la misión planteada para la escuela no funciona de manera adecuada, pues el ciudadano que egresa de la misma es distinto, y no de buen grado, al ideal que se espera.

Podemos aseverar que estamos ante la presencia de problemas entre grupos, donde las disyuntivas por sus marcadas diferencias también se manifiestan dentro de comunidad educativa, tales son un reflejo de lo que se observa y se vivencia en nuestra sociedad. Además, influye también el problema de las diferencias sociales, el cual está latente y parece ser una de las circunstancias que se añade como una dificultad para el proceso de paz.

A pesar de todo, aún existen una serie de afinidades que pueden ser empleadas como una vía alternativa para que entre los individuos puedan establecerse las relaciones sociales necesarias para el desarrollo de una la cultura de paz en la comunidad educativa.

A propósito de los valores, son una condición de relación que fortalece el vínculo entre las personas, además que su cultivo y fomento es una práctica que enriquece la cultura de paz, Fabelo (2004) nos indica que "los valores sólo pueden convertirse en fuentes motivacionales de los sujetos sociales y señalar una dirección y finalidad a su conducta si son subjetivamente asumidos por ellos, es decir, en tanto componentes de su conciencia" (p.39) y, uno de los valores necesarios para el fomento de la cultura de paz es el respeto, el cual al parecer brota con mayor facilidad entre las personas que tienen ideas en común, no sucediendo así generalmente cuando los individuos presentan desacuerdos.

Es notable que en algunos hogares se promueven los valores, pero sin embargo, en la mayoría no se desarrolla esta práctica de la manera adecuada, pues se afirma que no existe respeto y se abusa de la confianza depositada en los individuos, deteriorando el equilibrio de la cultura de paz esperada en la comunidad educativa.

Tomando en cuenta que la intención de los sujetos pacíficos es estar bien con quienes le rodean, en un entorno donde la agresividad, la violencia y la inseguridad está presente, las relaciones se ejecutan poniendo barreras de respeto como una actitud en defensa hacia las personas que le rodean, estableciendo un límite y dialogando sin prestar atención a la actitud que tomen. Como también existe una cierta inducción al rechazo hacia los sujetos respetuosos, 
amables y amigables con un hilo de desconfianza y recelo hacia los mismos, pero de alguna forma, son ellos quienes conviven en plenitud cuando pueden hacerlo.

A propósito de la tolerancia, esta se construye con la actitud de favorecer el crecimiento del otro, según la UNESCO (1994) "es un componente fundamental del respeto de los derechos humanos y para el logro de la paz. (...) consiste en reconocer a los demás el derecho a que se respete su persona e identidad" (p.12). Por lo tanto, reconociendo la realidad en que las discrepancias en cuanto a actitudes, opiniones, posiciones y pensamientos que generan enfrentamientos dentro de nuestra sociedad están presentes en la comunidad educativa, es necesario tomar en cuenta la tolerancia, que según la UNESCO (ob. cit.) "no es un fin, sino un medio. Es la calidad esencial mínima de las relaciones sociales que permite descartar la violencia y la coerción" (p.15).

Sin embargo, a pesar que entre los sujetos de estudio existe un conocimiento sobre la tolerancia, las circunstancias del contexto los conducen a lo contrario; y es más, probablemente podrían ser tolerantes fuera del mismo. Entonces, aquello denominado intolerancia está agravando los conflictos y es uno de los motivos que causan la alteración de la paz, pues se puede notar, que en el proceso educativo todos los participantes están afectados por la intolerancia, tanto los docentes, estudiantes y demás personal de la institución.

En la opinión de los sujetos de estudio, la intolerancia forma parte de la personalidad de cada persona $\mathrm{y}$, produce los conflictos que se generan entre los integrantes de la comunidad educativa, cuyo modelo es aprendido y, surge como consecuencia de no aceptar y diferir respecto a las ideas y pensamientos del otro, pues para la UNESCO (ob. cit.) “(...) sin tolerancia, la paz no es posible. Con tolerancia, es posible hacer realidad numerosas posibilidades humanas y sociales, y en particular la evolución de una cultura de paz" (p.15), aun mas cuando, en el ejercicio del derecho al libre pensamiento y el llevar a cabo las ideas, se debería evitar el empleo de métodos que perjudiquen a los integrantes de la comunidad educativa, como lo es el empleo de la violencia en todas sus formas. Es allí donde los docentes deben intervenir para que emerja la tolerancia necesaria entre los individuos que conforman nuestra sociedad.

\section{Sobre el liderazgo, la gerencia y otras habilidades sociales}

A propósito del liderazgo, este se concibe como habilidad social, pues como lo explica 
Gómez-Rada (2002) "es considerado como un fenómeno que ocurre exclusivamente en la interacción social; debe ser analizado en función de la relaciones que existen entre las personas en una determinada estructura social" (p.64), como en la familia, la comunidad y sus instituciones. De hecho, el liderazgo conduce a la cultura de paz generando beneficios para el desarrollo de los integrantes de la comunidad cuando el líder emplea su capacidad de significar tanto en el individuo como en el grupo; Viveros (2003), nos explica que es "el correcto ejercicio del mando, que se traduce en satisfacción del grupo de trabajo y de la empresa por haber cumplido con los objetivos encomendados". (p.3)

Por tal motivo, toda persona asume un liderazgo en determinado momento, desde liderarse a sí mismo, en la familia u hogar, en la comunidad educativa y en la sociedad, así lo explica la División de Organizaciones Sociales del Gobierno de Chile (2001)

La participación activa y comprometida se da sobre la base de una conducción por parte de los dirigentes, que permita y posibilite el protagonismo de sus miembros, de manera que realicen eficientemente sus acciones, por una parte y, por otra, que logren hacer realidad sus anhelos y cumplan los objetivos que toda la comunidad se haya propuesto. (p.11)

Sin embargo, se observa que la influencia de los líderes en la comunidad educativa es una de las circunstancias que alteran la cultura de paz tanto en la familia, las instituciones y la sociedad en general. Al respecto, tomemos a Gómez-Rada (2002), el cual nos explica que es "igualmente un hecho inequívoco que el comportamiento del líder influye en el desempeño de los subordinados, pero también es una realidad que el comportamiento de estos lo afecta". (p.74)

Por esa razón, el liderazgo que se ejerce en la actualidad, es una de las circunstancias que interfiere en el desarrollo pleno de una cultura pacífica tanto en los escenarios educativos y su comunidad, como en las diferentes esferas de nuestra estructura social; donde los líderes con sus acciones, comportamientos y actitudes son causa de su deterioro. Además, este modelo de liderazgo que se ha descrito y se tiene como referencia, se reproduce, afianzándose en la personalidad de nuestros ciudadanos.

Por tal motivo, debemos aprender a reconocer cuando el líder nos conduce hacia camino equivocado para el fomento de una cultura de paz y, cuando aquellos a cargo en la ejecución de sus labores y la influencia que tienen, nos alejan de ella. Así como también, dadas las circunstancias, es necesario asumir un rol protagónico y conducir a la juventud hacia una 
cultura pacífica que nos permita obtener soluciones positivas a los conflictos que se han generado socialmente, haciendo una convocatoria a los ciudadanos cuya motivación interior a tomar las riendas, debido a su experiencia, preparación y capacitación tanto en lo académico, lo social y lo familiar, puedan alcanzar el logro de tal objetivo.

A propósito de la gerencia, esta es concebida como una habilidad social pertinente a la administración de una organización, donde la ética profesional y la moral son los pilares fundamentales para su ejecución al momento de dirigir a sus integrantes hacia una cultura de paz. Alvarado (2011) expresa acerca de la gerencia que:

Cuanto mayor sea la complejidad del ámbito en el cual una empresa opera, más necesario será que esa persona tenga internamente individuos capaces de colaborar con los otros, de motivarlos, de hacer funcionar un proyecto: ellos son el collar que mantiene junto el grupo y que hace posible una sana y productiva interacción interna. (p.16)

Sin embargo, en la realidad observada, se aprecia que la gerencia es una necesidad por atender, puesto que "la capacidad de hacer trabajo en equipo (...) característica más importante y buscada en cualquier nivel al interno de una organización" (p.16), según Alvarado (ob. cit.), no es eficiente para el curso positivo de una cultura de paz.

Por esta razón, es necesario que la organización se involucre, indague y dedique sus esfuerzos en el proceso de solventar los conflictos presentes. Berghe (2011) manifiesta que "es despertar el interés de sus colaboradores, con una mente abierta al cambio, que permita y propenda porque sus colaboradores presenten innovaciones individuales o en grupo, por lo tanto es necesaria la cooperación de todos los empleados". (p.6)

A propósito de las habilidades sociales, éstas se refieren a la calidad de la relación y comunicación entre los sujetos, de tal manera se considera que para obtener una cultura de paz en el entorno cada persona debe saber interactuar con el resto, transmitir sus ideas considerando respeto hacia sí mismo y, considerar que la comunicación es una alternativa para resolver los conflictos que se presenten.

A propósito de la autoestima, Gil (2001) destaca que “(...) es la suma de la confianza y el respeto que debemos sentir por nosotros mismos y refleja el juicio de valor que cada uno hace de su persona para enfrentarse a los desafíos que presenta nuestra existencia" (p. 10) como lo es establecer una cultura de paz necesaria. 
A propósito de la comunicación, se observó que dentro del entorno los sujetos pueden mantener un diálogo acerca de cualquier tema teniendo en cuenta un respeto recíproco, lo cual es lo ideal y más propicio para que pueda desarrollarse una cultura de paz al momento de transmitir sus puntos de vista. Así como también los sujetos tratan de adecuarse o adaptarse a su interlocutor para hacerle llegar sus ideas, pero sin embargo, en las relaciones que se entablan suelen hacerse prejuicios acerca de las personas que aún no se conocen como consecuencia de las diferentes experiencias que se viven a diario, como mecanismos de defensa para la integridad personal.

Se pudo notar un desarrollo de la comprensión del lenguaje no verbal y la empatía al momento de reconocer en la otra persona sus estados de ánimo, así como también, una forma de interacción entre los sujetos de estudio, que es tentar las emociones: una forma de comunicación, con o sin intención de agredir, pero que bien puede no ser interpretada de la manera adecuada porque el mensaje no está completo, o también, interesa saber cuál es la interpretación, como también existe una estructura donde los pensamientos y las emociones están en conjunto susceptibles a una señal externa, pues como expresa un actor social que cuando observa a alguien, y ese sujeto se imagina lo que está pensando, puede haber un problema.

A propósito de la resolución de conflictos, esta suele hacerse entre sujetos afines o que pertenezcan a un mismo grupo, pues la comunicación es de mejor calidad y más comprensible, Novarra (2007) dice que: "estar bien con uno mismo y con los demás significa reconciliarse con la posibilidad de que los conflictos no solo formen parte de nuestra vida corriente, sino que sean uno de los momentos de profundo aprendizaje creativo" (p. 10)

\section{Sobre los intereses}

A propósito de los intereses particulares, estos representan un factor relevante que altera la cultura de paz, en base a estos se ocasionan conflictos y se juega al caos para obtener beneficios como lo es obtener comodidad y poco trabajo, conduciendo a los estudiantes al disentimiento, al no razonamiento y la no equitatividad haciendo uso de la complicidad para lograr sus objetivos. 
En resumen, la estructura observada nos devela que las habilidades sociales presentes en los actores sociales de la comunidad educativa, como lo son la tolerancia, el liderazgo y la gerencia, se encuentran dispersas negativamente tanto en la labor docente como en la familia, generando una brecha en la construcción de la convivencia pacífica tomando en cuenta la carga de agresividad y violencia además los intereses presentes.

\section{REFLEXIONES FINALES}

Se debe reconocer que la paz se alimenta y sustenta con las acciones y actitudes labradas en el convivir desde el entorno familiar hasta el social, las cuales, generan una mejor calidad de vida en actividades de mayor provecho para el hombre, donde para el desarrollo de una cultura de paz, debe enfocarse las habilidades sociales de los integrantes de la comunidad educativa en impulsar la tolerancia, el liderazgo y la gerencia basada en los valores que conduzcan a la construcción de una convivencia pacífica socioeducativa haciendo énfasis desde las familias y la labor docente.

Por tal motivo, es necesario estimular a todos los integrantes de la comunidad para que promuevan un ambiente de convivencia pacífica mediante su fomento desde la familia, tomando en cuenta los valores de libertad, justicia, tolerancia y respeto, como también, se debe aunar esfuerzos y organizarse para asumir la conflictividad cotidiana generada por los focos de agresividad y violencia presente con una actitud positiva que promueva la cultura de paz.

Así como también, se necesita lograr una gerencia y administración educativa con la moral y la ética profesional como pilares fundamentales, que se involucre en el proceso de solventar los conflictos que se presentan dentro de la comunidad para el desarrollo de una convivencia plena, y de la misma manera, fomentar un liderazgo capaz de conducir a las instituciones hacia un camino cónsono a la cultura de paz, por medio del cual, los integrantes realicen eficientemente sus labores para el logro de sus sueños y anhelos cumpliendo con los objetivos que toda la comunidad se haya propuesto.

\section{REFERENCIAS}

Alvarado, F. (2011). Gerencia del siglo XXI. [Documento en línea]. Disponible: http://www.consejeriaempresarial.com/publicaciones/La\%20gerencia\%20del\%20Siglo\%20 XXI.pdf. [Consulta: 2013, Septiembre 22]

Berghe, E. (2011). Gerencia del futuro. [Documento en línea]. Disponible: 
http://www.cpae.gov.co/userfiles/2_LA\%20GERENCIA\%20DE\%20HOY\%20Y\%20DEL\% 20FUTURO.pdf. [Consulta: 2013, Septiembre 22]

Buxarrais, M. (1997). La formación del profesorado en educación en valores. Propuesta y materiales. Bilbao: Descleé De Brouwer.

División de organizaciones sociales. (2001). Liderazgo. Gobierno de chile.

Fabelo, J. (2004). Los valores y sus desafíos actuales. [Documento en línea]. Disponible: http://educarteoax.com/pedagogizando/descargas/otros/

los_valores.pdf. [Consulta: 2013, Septiembre 23]

Gil, R. (2001). Manual para tutorías y departamentos de orientación. Bilbao: Monografías Escuela Española.

Gil-Verona, J. (2002). Anales de psicología. [Documento en línea]. Disponible: http://www.um.es/analesps/v18/v18_2/07-18_2.pdf. [Consulta: 2013, Septiembre 23]

Goleman, D. (1995). La inteligencia emocional. Buenos Aires: Javier Vergara Editor.

Gómez-Rada, C. (2002). Liderazgo: conceptos, teorías y hallazgos relevantes. [Documento en línea]. Disponible: http://www.uelbosque.edu.co/sites/default/

files/publicaciones/revistas/cuadernos_hispanoamericanos_psicologia/volumen2_numero2/artic ulo_5.pdf. [Consulta: 2013, Septiembre 23]

González, F. (2009). Manual de estrategias pedagógicas sobre educación para la paz en el nivel preescolar dirigido a los docentes de las escuelas Fe y Alegría del Municipio Iribarren Estado Lara. Barquisimeto: Universidad Pedagógica Experimental Libertador.

Martínez Miguélez, M. (1996). Comportamiento humano. México: Editorial Trillas.

Martínez Miguélez, M. (1997). El Paradigma Emergente. Hacia una Nueva Teoría de la Racionalidad Científica. México: Editorial Trillas.

Martínez Miguélez, M. (2006). Ciencia y arte en la metodología cualitativa. México: Editorial

Martínez Miguélez, M. (2008). Epistemología y metodología en las ciencias sociales. México: Editorial Trillas.

Molano, O. (2011) Participación transformadora del liderazgo docente como expresión universitaria de una cultura ciudadana para la paz. Barquisimeto: Universidad Pedagógica Experimental Libertador.

Morales, M. (2007). Ser persona y relacionarse. Habilidades cognitivas y sociales y crecimiento moral. Madrid: Narcea Ediciones. 
Novarra, D. (2007). Educación socioafectiva. 150 actividades para conocerse, comunicarse y aprender de los conflictos. Madrid: Narcea, S.A. de ediciones.

OMS. (2002). Informe mundial sobre la violencia y la salud. Washington, DC: OPS

Pérez Serrano, G. (1998). Investigación cualitativa: retos e interrogantes. Madrid: La Muralla.

Rusque, A. M. (2007). De la diversidad a la unidad en la investigación cualitativa. Caracas: Vadell Hermanos.

UNICEF. (2002). Unicef va a la escuela para construir una cultura de paz y solidaridad. [Documento en línea]. Disponible: http://www.unicef.org/argentina/spanish/ar_insumos_educvaescuela4.PDF [Consulta: 2013, Septiembre 17]

UNESCO. (1994). La tolerancia, umbral de la Paz. Guía didáctica de educación para la Paz, los derechos humanos y la democracia. [Documento en línea]. Disponible: http://www.ocse.org.mx/pdf/130_Tolerancia.pdf. [Consulta: 2013, Septiembre 23]

UNESCO. (2002). Manual de la conferencia general. Paris. Unesco.

Viveros, J. A. (2003). Liderazgo, comunicación efectiva y resolución de conflictos. Santiago: Oficina Internacional del Trabajo.

Yegres, M. (1999). Ética y formación docente. Caracas: Universidad Pedagógica Experimental Libertador. 